\title{
Tailoring incoming shear and turbulence profiles for lab-scale wind turbines
}

\author{
R. Jason Hearst ${ }^{1,2}$ Bharathram Ganapathisubramani $^{1}$ \\ ${ }^{1}$ Engineering and the Environment, University of Southampton, Southampton, UK, SO17 1BJ \\ ${ }^{2}$ Department of Energy and Process Engineering, Norwegian University of Science and Technology, Trondheim, Norway, N-7491
}

\section{ABSTRACT}

An active grid is used to generate a variety of turbulent shear profiles in a wind tunnel. The vertical bars are set to flap through varying angles across the test-section producing a variation in the perceived solidity, resulting in a mean shear. The horizontal bars are used in a fully random operational mode to set the background turbulence level. It is demonstrated that mean velocity profiles with approximately the same shear can be produced with different turbulence intensities and local turbulent Reynolds numbers based on the Taylor microscale, $\lambda$. Conversely, it is also demonstrated that flows can be produced with similar turbulence intensity profiles but different mean shear. It is confirmed that the length scales and dynamics, the latter being assessed through the velocity spectra and probability density functions, do not vary significantly across the investigation domain. Such flows are of particular relevance for studies investigating the effect of in-flow conditions on obstacles where these studies wish to decouple the effects of turbulence intensity and mean shear, a feat previously unattainable in experimental facilities. Given that the power output of wind turbines is known to be a function of both mean shear and turbulence intensity, the experimental methodology presented herein is invaluable to the wind turbine model testing community who, at present, cannot exert such control authority over the in-flow conditions. Copyright (C) 2017 John Wiley \& Sons, Ltd.

\section{KEYWORDS}

turbulence, shear flow, simulated atmospheric flows, active grid, wind tunnel, wind turbine

\section{Correspondence}

Email: g.bharath@southampton.ac.uk

Received... 


\section{INTRODUCTION}

The majority of flows of engineering interest are turbulent in nature. A major subset of these are also sheared. These flows are of particular relevance for studies involving wind turbines as in practice turbines operate within sheared flows that naturally occur in the atmospheric boundary layer and the wakes of other turbines. In addition, this shear is not necessarily constant, nor is it universal. Depending on weather patterns, local topography and the layout of a wind farm, a wind turbine may experience a variety of different incoming shear and turbulence intensity profiles. Simulating these varied conditions in an experimental facility is incredibly difficult, and as such experimentalists have struggled to decouple the effects of turbulence intensity and shear in wind tunnel tests. On a fundamental fluids level, the specific case of homogeneous turbulent shear flow (approximated in wind tunnels as uniformly sheared flow) is also of great theoretical interest because it is one of the simplest fundamental flows that can be investigated [1]. Despite the frequency of occurrence of turbulent shear flows, there are relatively few methods of producing them in experimental facilities, particularly at local turbulent Reynolds numbers that are representative of real turbulent flows encountered by wind turbines; measurements of the Taylor microscale Reynolds number, $R e_{\lambda}$ (defined fully later), in the atmospheric boundary layer are of order 1000 and above [2,3], which is significantly higher than $R e_{\lambda} \lesssim 200$ readily produced in wind tunnels with passive grids [4, 5, 6].

For a wind turbine, the coefficient of power, as defined by the International Electrotechnical Commission (IEC) standard for measuring wind turbine performance [7], is

$$
C_{P}=\frac{P}{\frac{1}{2} \rho U_{H}^{3} A},
$$

where $P$ is the power output of the turbine, $\rho$ is the air density, $U_{H}$ is the mean velocity measured at the hub height, and $A$ is the swept area of the turbine blades. The denominator of (1) is the kinetic energy flux through the turbine's swept area,

$$
\mathcal{K}_{H}=\frac{1}{2} \rho U_{H}^{3} A
$$

which is proportional to the power output of the turbine for a constant $C_{P}$. However, a limitation of the above definitions is that they functionally assume the velocity across the entire turbine is constant and equal to $U_{H}$. To this end, Wagner et al. [8] presented measurements from met masts at Høvsøre and identified that incoming profiles with velocities between $6 \mathrm{~m} / \mathrm{s}$ and $8 \mathrm{~m} / \mathrm{s}$ at $80 \mathrm{~m}$ could have over 170 different profiles. This led them to develop a discretised integral quantity that incorporated the effects of wind shear, which they referred to as the 'equivalent wind speed',

$$
U_{\mathrm{eq}, \mathrm{S}}=\left[\frac{1}{A} \sum_{i} U_{i}^{3} A_{i}\right]^{1 / 3}
$$

where the subscript $\cdot_{i}$ denotes a quantity measured at a given position in the velocity profile. An example of how the segments might be broken down is provided in Figure 1. This quantity was directly related to the discretised kinetic energy 

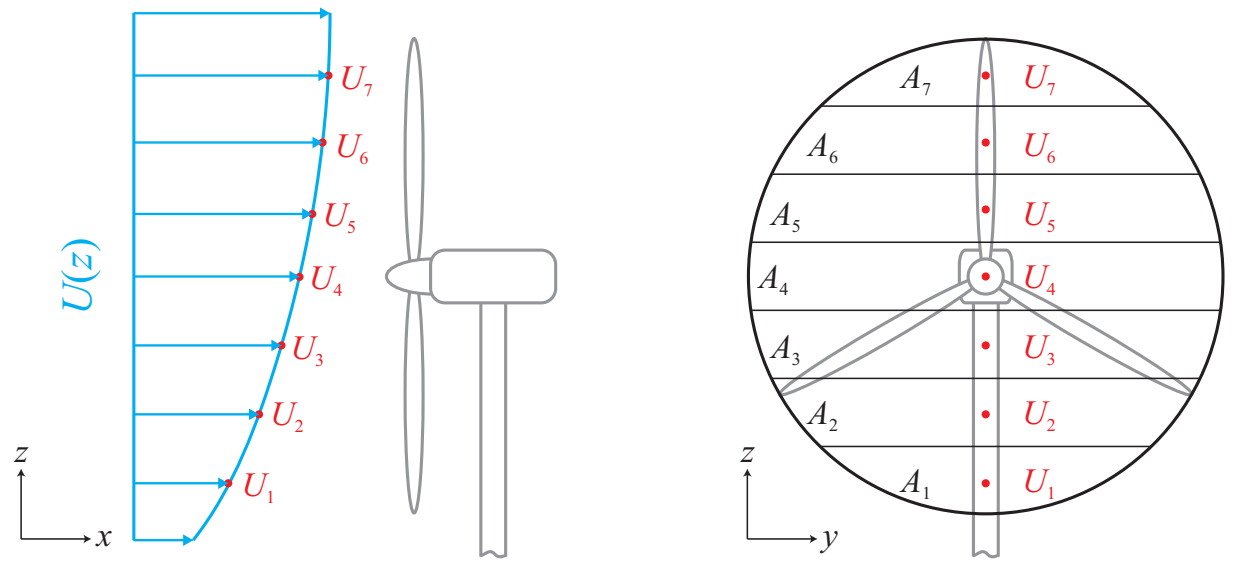

Figure 1. Example schematic of a discretised incoming velocity field and swept area break-down for a wind turbine. The dots represent measurement positions.

flux through the turbine's swept area which is more accurately represented by [9],

$$
\mathcal{K}_{S}=\frac{1}{2} \rho \sum_{i} U_{i}^{3} A_{i}
$$

Roughly, Wagner et al. [9] found that $0.85 \lesssim \mathcal{K}_{S} / \mathcal{K}_{H} \lesssim 1.1$ for their measurements. They also found that $U_{\text {eq,S }}$ was able to approximately collapse power curves measured in different levels of wind shear. This idea was expanded to include the effects of turbulence intensity and wind direction by Choukulkar et al. [10], who gave the equivalent wind speed as

$$
U_{\mathrm{eq}}=\left\{\frac{1}{A} \sum_{i} U_{i}^{3}\left[1+3\left(\frac{u_{i}^{\prime}}{U_{i}}\right)^{2}\right]\left[1-\frac{\Phi^{2}}{2}-\frac{\left\langle\phi_{i}{ }^{2}\right\rangle}{2}\right]^{3} A_{i}\right\}^{1 / 3},
$$

where $u^{\prime}$ is the standard deviation of the velocity fluctuations, $\Phi$ is the mean incoming angle of the flow, and $\left\langle\phi^{2}\right\rangle$ is the variance of the incoming angle fluctuations. These equivalent wind speed estimations have been approximately validated in the field, but a full understanding of the effect of shear and turbulence intensity on the power output of a wind turbine is still a topic of contemporary research. As such, developing controllable velocity fields in a wind tunnel where the parameters in (5) can be varied independently is of great interest.

The need for the above formulations arose because it was clear that the energy content was vastly different in flows with approximately the same velocity at turbine hub height but different shear and turbulence distributions $[8,9,10]$. The shear of the incoming flow is often parameterised in wind turbine flows with a power-law,

$$
\frac{U}{U_{r}}=\left(\frac{z}{z_{r}}\right)^{\alpha}
$$

where $z$ is the wall-normal (vertical) direction and the subscript ${ }_{r}$ denotes a quantity measured at a reference position. For a neutral atmospheric boundary layer $\alpha \approx 0.143$, and for the constant incoming velocity case $\alpha=0$. Wagner et al. [9] 
found that the greatest deviations from $\mathcal{K}_{S} / \mathcal{K}_{H} \approx 1$ typically occurred for profiles that were not well described by (6), but were nonetheless prevalent in the incoming flows. Dimitrov et al. [11] showed that $\alpha$ was dependent on the mean wind velocity and turbulence intensity. They found that the incoming velocity profile could vary in the range $-0.20 \lesssim \alpha \lesssim 0.35$. With respect to the turbulence intensity, the data presented by Mücke et al. [12] from GROWIAN demonstrated that the turbulence intensity of the incoming flow could reach $40 \%$ at that site, but typically ranged from $5 \%$ to $15 \%$. Thus, the incoming flow for a wind turbine is described by a large parameter space of turbulent shear flows, and not only a canonical neutrally buoyant atmospheric boundary layer.

Over the years, turbulent shear flows in experimental facilities have been generated using a handful of methodologies. The most intuitive methodology is to use the boundary layer naturally developing on one of the facility walls. A typical addition to this approach, used to produce atmospheric-like conditions, is to place a series of spires at the inlet of a wind tunnel test-section $[13,14,15,16]$. Some examples of model wind turbine studies that use the boundary layer to generate shear are those of Bossuyt et al. [17] and Chamorro and Porté-Agel [18]. Bossuyt et al. [17] placed a model wind farm of porous discs in a turbulent boundary layer that was tripped at the start of their test-section, while Chamorro and PortéAgel [18] compared cases for their model turbine placed in the boundary layer forming over a smooth wall and a rough wall. The general limitations of using the boundary layer to produce the shear profile are the number of profiles that can be produced, typically centered about $\alpha=0.143$, and the range of turbulence intensities that can be realised, typically between $7 \%$ and $13 \%[13,18,19]$. To produce homogeneous turbulent shear flows, porous plates with varying solidity or cascading meshes with different solidity, both followed by flow straighteners, have been used [1, 20]. However, both these techniques are limited in that one, very complicated and tailored, set-up results in a single shear profile without much variability in Reynolds number or velocity profiles. The relevant Reynolds number for a turbulent flow is that based on the Taylor microscale, and given by

$$
R e_{\lambda}=\frac{u^{\prime} \lambda}{\nu}
$$

where $\lambda$ is the Taylor microscale and $\nu$ is the kinematic viscosity.

The advent of active grids has ushered in a new era of turbulent flow testing in wind tunnels. These devices, popularised by the original study of Makita [21], offer increased flexibility in the turbulent flows generated in wind tunnel facilities, allowing for some degree of control over the produced turbulence intensity, length scales, and $R e_{\lambda}$ [22, 23]. In general, these devices consist of a series of wings mounted to rods that are actuated in different random patterns by stepper motors. A schematic and image of the University of Southampton active grid are provided in Figure 2. The active grid wings can be a variety of geometries, but are typically flat square plates, perforated flat square plates, or flat circular plates; see [23] for discussion of the effect of the various geometries. An active grid is employed to produce wind tunnel turbulence by placing it at the inlet of the test-section. Turbulence is generated as flow passes through the transient blockage. If a random protocol is used to actuate the rods of the active grid, then the flow becomes approximately homogeneous in transverse planes sufficiently far downstream; typically $~ 30 M$ or so, where $M$ is the mesh length (spacing) between rods of the grid [22, 23]. The various rods of the grid can be operated with different rotational rates $(\omega)$, and cruise times between 


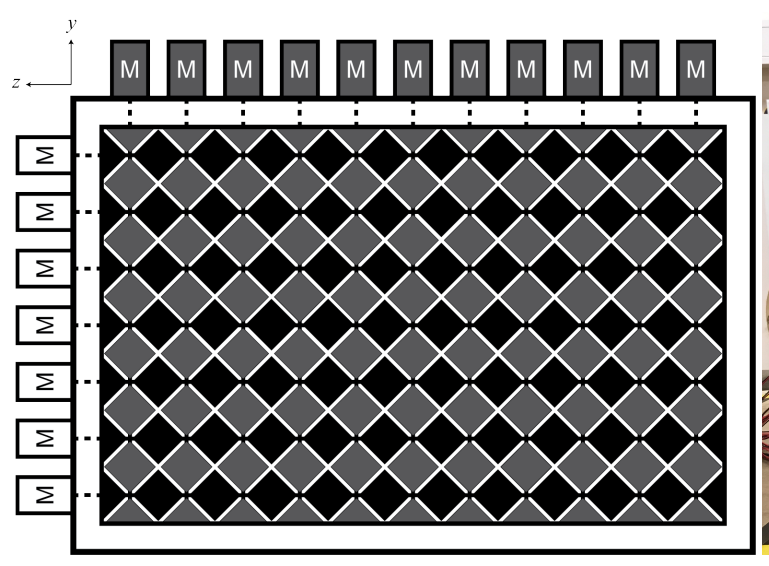

(a)

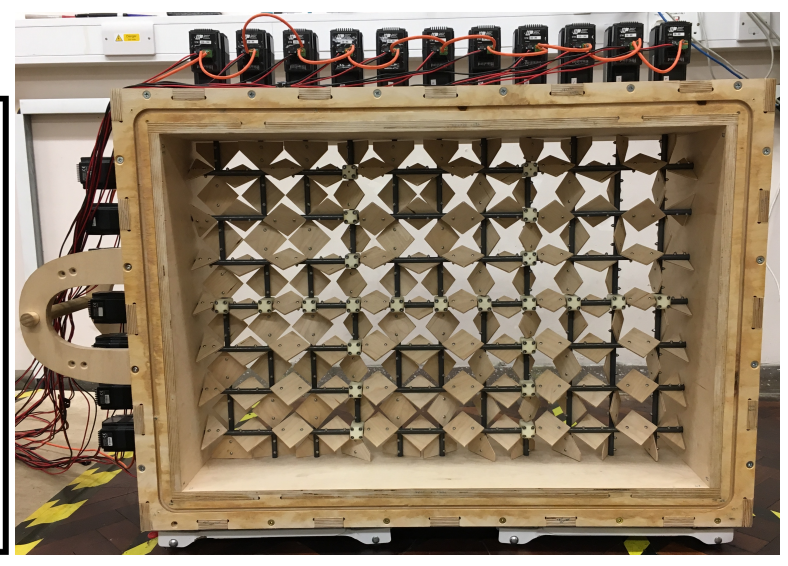

(b)

Figure 2. (a) Schematic of the active grid used in the present study. Grey wings and motors identify the vertical rod system that is responsible for the shear profile. Black wings and white motors represent the horizontal rod system that is responsible for the turbulence level. (b) Picture of the active grid used in the present study outside of the wind tunnel.

updating motor commands $(t)$ to vary the characteristics of the turbulence. These devices have become quite popular and there are several of them around the world. The most advanced grid is likely that described by Bodenschatz et al. [24], where each wing is actuated by its own motor; typically the motion of all wings along a single rod is coupled because the rod is actuated by a single motor. The grid with the largest number of elements across a wind tunnel is that of Hearst and Lavoie [23], who have also performed the most detailed parametric study on the produced turbulence to date.

In addition to producing approximately homogeneous free-stream turbulent flows, active grids can also be used to generated bespoke shear profiles. This idea was pioneered by Shen and Warhaft [25] who placed an active grid upstream of a variable density mesh followed by flow straighteners. Using this configuration they produced an approximately homogeneous shear flow with $R e_{\lambda} \sim 1000$, however, the set-up still necessitated the addition of meshes and flow straighteners downstream of the grid. Cal et al. [15] used a series of spires in conjunction with an active grid to increase the turbulence intensity and Reynolds number of their incoming flow to a model wind farm. The drawback of this particular set-up was that the shear was predominantly governed by the spires and the boundary layer. Cekli and van der Water [26] demonstrated that active grids could produce both homogeneous shear turbulence and a synthetic atmospheric boundary layer. This was achieved by setting the various rods of their active grid into flapping motions with different initial offsets. Thus, they produced variable porosity across their test-section inlet with their active grid directly rather than using variable porosity meshes and flow straighteners downstream. Later, Hearst el al. [27] took a different approach to generating bespoke shear profiles, by using an active grid to produce free-stream turbulence, which in turn altered the mean geometry of the turbulent boundary layer forming over a plate; see also [28] for further details on this flow. Using this approach, Hearst et al. [27] were able to investigate the wake of a turbulent boundary layer-immersed cube under differing turbulence intensities but the same non-dimensional shear, thus explicitly decoupling shear from turbulence intensity for the first time. However, this approach was limited to the shear levels and velocity profiles produced by the wall, and the authors had to 


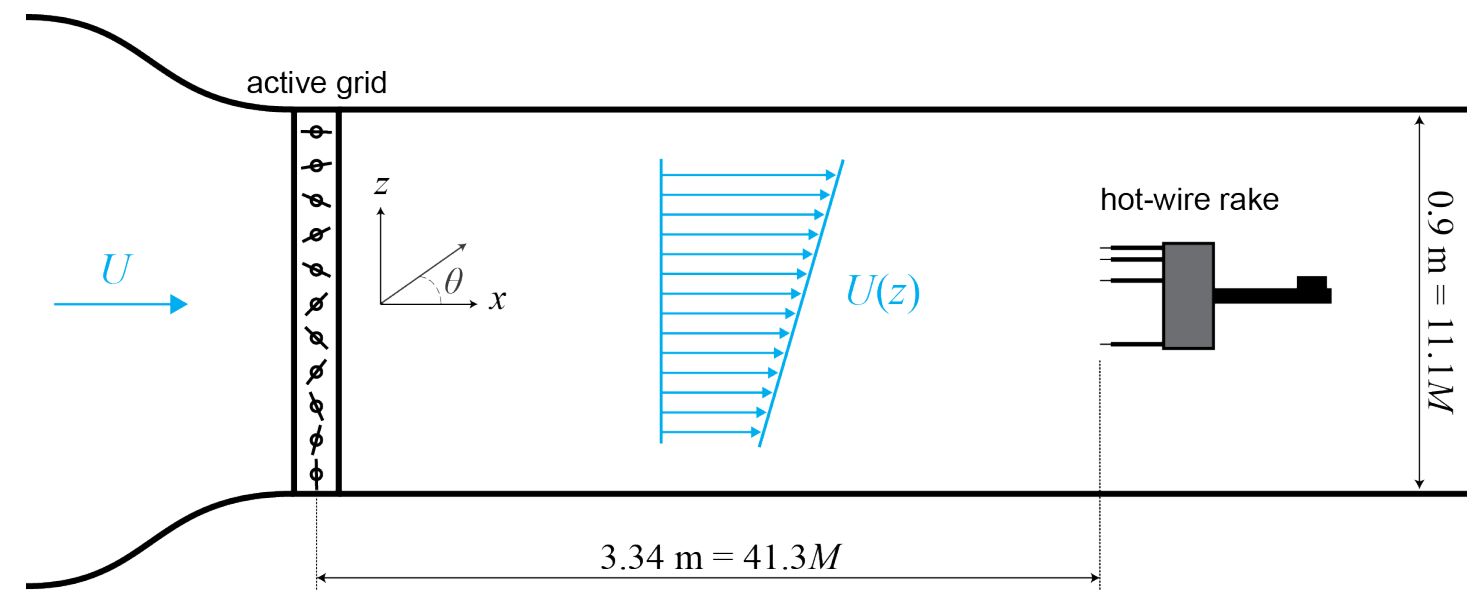

Figure 3. Schematic of the experimental set-up illustrating a view from beneath the wind tunnel (not to scale). The wing at the top of the figure is at $\theta=0^{\circ}$, and the wing at the bottom of the figure is at $\theta=90^{\circ}$.

change the size of their model between test cases to keep the non-dimensional shear constant relative to their obstruction. Furthermore, the size of their investigation domain was limited to the boundary layer produced in their facility $(\sim 0.1 \mathrm{~m})$. Thus, there is a need to produce flows with similar flexibility over a wider region in the wind tunnel.

The present study returns to the technique of Cekli and van der Water [26] and explores the produced flows in greater detail. While Cekli and van der Water [26] demonstrated that one could generate a homogeneous shear flow without the need of additional variable porosity meshes or flow straighteners, they did not explore producing the same flow at different $R e_{\lambda}$ or turbulence intensities. This is of particular interest for wind turbine flows where the same shear profile may present consistently while the turbulence levels may vary. This work also explores the generation of other flow profiles that allow for the ultimate goal of being able to produce similar turbulence levels with markedly different shear profiles. Such flows would allow for the opposite comparison (turbulence is fixed but shear is changed), which is presently unattainable in experimental facilities. Understanding how to generate these flows and then employing them to investigate the impact of shear and turbulence intensity on wind turbines is of great interest to the community and would allow for the various terms in (5) to be varied independently.

\section{EXPERIMENTAL PROCEDURE}

The active grid used for this study is the same as that employed in Ref. [27, 28]. A full description of the device is given by Dogan et al. [28], however, we summarise the relevant details here. The grid is composed of a $11 \times 7$ array of bars to which flat solid square wings are mounted (Figure 2). The mesh length, $M$, of the grid is $81 \mathrm{~mm}$. Each rod of the grid is actuated by a separate stepper motor. Thus, all wings along a single axis are coupled, and there are 18 degrees-of-freedom. The stepper motors are controlled via a computer. The grid was positioned at the inlet of the wind tunnel test-section. The wind tunnel itself is the suction-type tunnel located at the University of Southampton that has a $0.9 \mathrm{~m} \times 0.6 \mathrm{~m} \times 4.5 \mathrm{~m}$ test-section. A schematic of the experimental configuration is provided in Figure 3. 
Table I. Flapping amplitude for the vertical bars used to generate the shear profiles. All values are in degrees and are relative to the initial position parallel to the mean flow.

\begin{tabular}{c|ccccccccccc} 
& \multicolumn{10}{c}{ Rod Position $(z)$} \\
\cline { 2 - 13 } Shear Mode & $-5 M$ & $-4 M$ & $-3 M$ & $-2 M$ & $-1 M$ & 0 & $1 M$ & $2 M$ & $3 M$ & $4 M$ & $5 M$ \\
\hline 1 & 90.0 & 81.0 & 72.0 & 63.0 & 54.0 & 45.0 & 36.0 & 27.0 & 18.0 & 9.0 & 0 \\
2 & 90.0 & 66.7 & 49.4 & 36.6 & 27.2 & 20.1 & 14.9 & 11.1 & 8.2 & 6.1 & 4.5 \\
3 & 90.0 & 45.0 & 39.4 & 33.8 & 28.1 & 22.5 & 16.9 & 11.3 & 5.6 & 0 & 9.0 \\
4 & 4.5 & 36.0 & 63.0 & 54.0 & 45.9 & 45.0 & 27.0 & 13.5 & 9.0 & 0 & 0
\end{tabular}

Table II. Random rotation parameters for the horizontal bars used to manipulate the turbulence level.

\begin{tabular}{c|cc|cc|cc|l}
\multirow{2}{*}{ Turb. Mode } & \multicolumn{2}{|c|}{$t[\mathrm{~s}]$} & \multicolumn{2}{c|}{$\omega[\mathrm{Hz}]$} & \multicolumn{2}{c|}{$a[\mathrm{~Hz} / \mathrm{s}]$} & \multirow{2}{*}{} \\
\cline { 2 - 7 } & $\min$ & $\max$ & $\min$ & $\max$ & $\min$ & $\max$ & \\
\hline A & - & - & - & - & - & - & bars stationary at $\theta=0^{\circ}$ \\
B & 0.05 & 2.29 & 5.00 & 15.00 & 50 & 200 & low turbulence \\
C & 0.05 & 2.82 & 1.00 & 3.00 & 50 & 200 & medium turbulence \\
D & 0.05 & 2.24 & 0.25 & 0.75 & 50 & 200 & high turbulence
\end{tabular}

The coordinate system used in this study is $x$ is the streamwise direction, $y$ is the vertical direction, and $z$ is the horizontal direction. The standard decomposition of time-series velocities into a mean, $U$, with a fluctuation, $u$, is employed. A prime, e.g., $u^{\prime}$, is used to denote the standard deviation of the signal.

Shear was produced in the horizontal, $z$, direction because the active grid has more degrees-of-freedom across this direction. The shear was set by adjusting the motion of the vertical bars. All the wings attached to vertical bars were initially set parallel to the mean flow direction, $\theta=0^{\circ}$, and then oscillated through different angles, $\theta$, depending on their horizontal position across the wind tunnel. The wings upstream of the high velocity region of the shear profile would oscillate through low angles, e.g., $\theta= \pm 9^{\circ}$, while the wings upstream of the low velocity region would oscillate through high angles, e.g., $\theta= \pm 90^{\circ}$. This differs marginally from the technique of Cekli and van der Water [26], who adjusted the initial position of their wings to alter the blockage and then oscillated each wing about this offset. In the present study, four different 'shear modes' were used, as detailed in Table I. Taking shear mode 1 as an example, the wings at $z / M=+5$ are held constant at the minimum blockage position $\theta=0^{\circ}$, and the angle of oscillation for each set of wings varies linearly across the wind tunnel until $\theta= \pm 90^{\circ}$ at $z / M=-5$. The input for shear mode 2 is a log profile, and shear modes 3 and 4 were arrived at by trial-and-error. The objective of the trial-and-error procedure was simply to produce a series of profiles with different $\partial U / \partial z$ that were representative of flows encountered by wind turbines. The maximum reliable update speed to the grid was $20 \mathrm{~Hz}$, and as such this represents the maximum flapping frequency of the grid, with the flapping frequency instantaneously taking-on any random value below this maximum. Flapping commands were sent to all shear generating wings at the same time, but with the order of actuation assigned randomly. The initial flapping direction was always set to alternate between adjacent vertical rods. Without the randomisation of the flapping order and the forced alternating pattern between rods, it was found that a distinct peak was visible in the produced velocity spectra that was related to the flapping frequency of the shear mode wings. A sample flapping sequence for shear mode 1 is presented on the right side of Figure 4.

The four shear modes were supplemented with four 'turbulence modes'. The turbulence modes describe the operational parameters given to the horizontal bars. The horizontal bars were used to adjust the background turbulence intensity for 


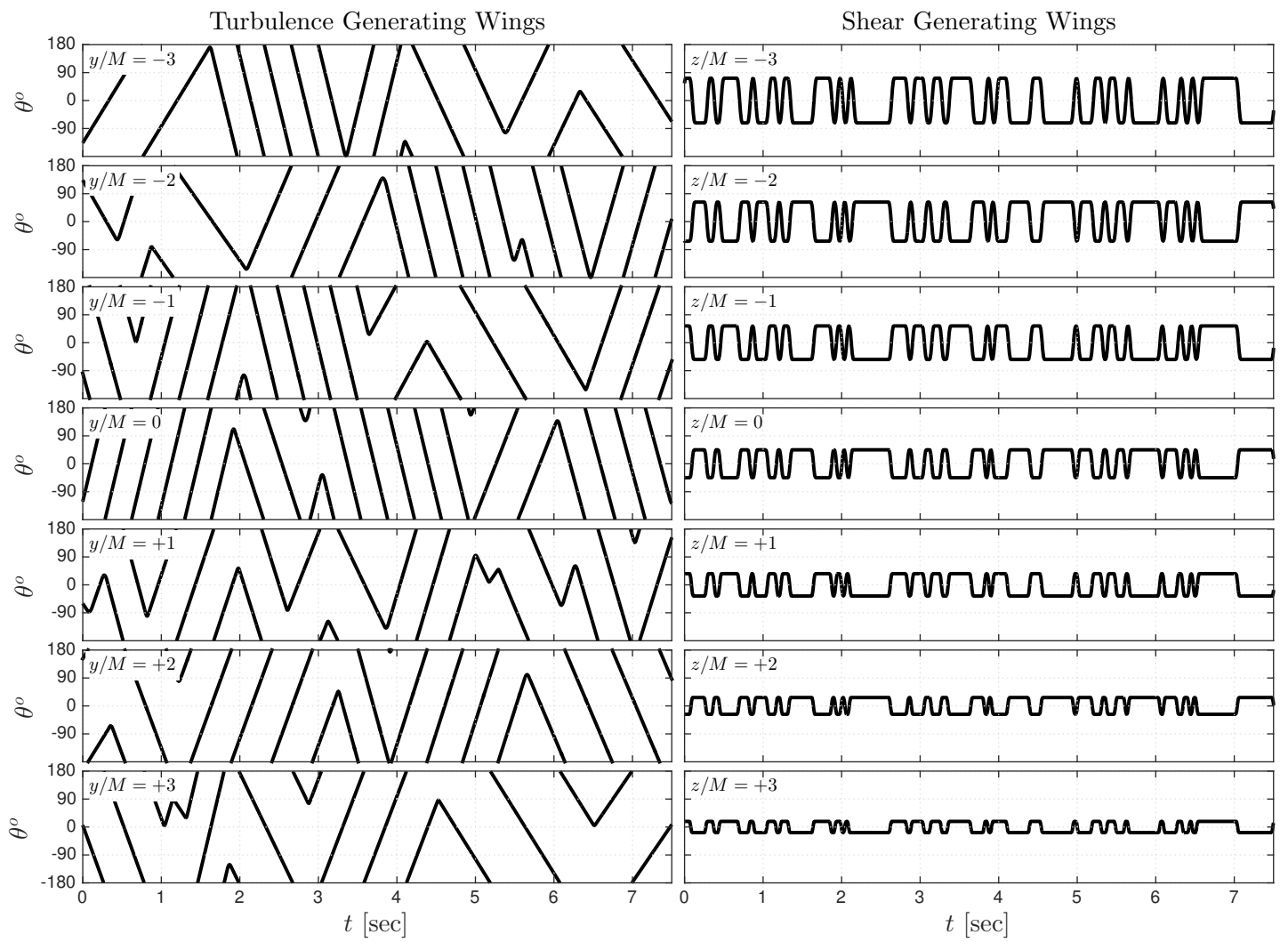

Figure 4. Sample time-series of central $7 \times 7$ rods for actuation sequence $1 \mathrm{C}$.

each of the shear modes. Turbulence mode 'A' is used to refer to the case where the horizontal bars were held fixed, while $\mathrm{B}, \mathrm{C}$ and D represent low, medium and high turbulence intensity modes, respectively. These modes differ primarily in the range of the random rotational velocities fed to the grid. In accordance with previous studies, low rotational velocity of the wings $(\omega)$ was found to produce high turbulence intensity, and high $\omega$ produced low turbulence intensity [23]. The horizontal rods were actuated with the fully random mode described in [23], where each rod was given a randomised signal of cruise time $(t)$, rotational velocity $(\omega)$, and rotational accelerations $(a)$. For the horizontal bars that were responsible for the background turbulence, the cruise time refers to the time between new commands, i.e., if a bar receives a command to rotate at $\omega=6 \mathrm{~Hz}$ for $t=1 \mathrm{~s}$, then it will complete six complete rotations in one second before receiving a new command. This contrasts with the flapping motion of the vertical bars that never complete a full rotation, but instead flap about $\theta=0^{\circ}$. The random distribution used for the horizontal bars was a top-hat (equal probability to all values in the set). The parameters used for the turbulence modes are provided in Table II. Figure 5 compares the B, C, and D turbulence actuation protocols. The primary difference is the change in $\omega=\partial \theta / \partial t$ which results in fewer full revolutions for the same period. Note that because the turbulence generating wings can rotate fully about their central axes, $\theta$ wraps from $-180^{\circ}$ to $+180^{\circ}$ and vice-versa.

The four shear modes and four turbulence modes were combined to generate 16 distinct turbulent shear flows. These flows were named according to their generation mode, e.g., when the grid was operating with shear mode ' 1 ' and turbulent 


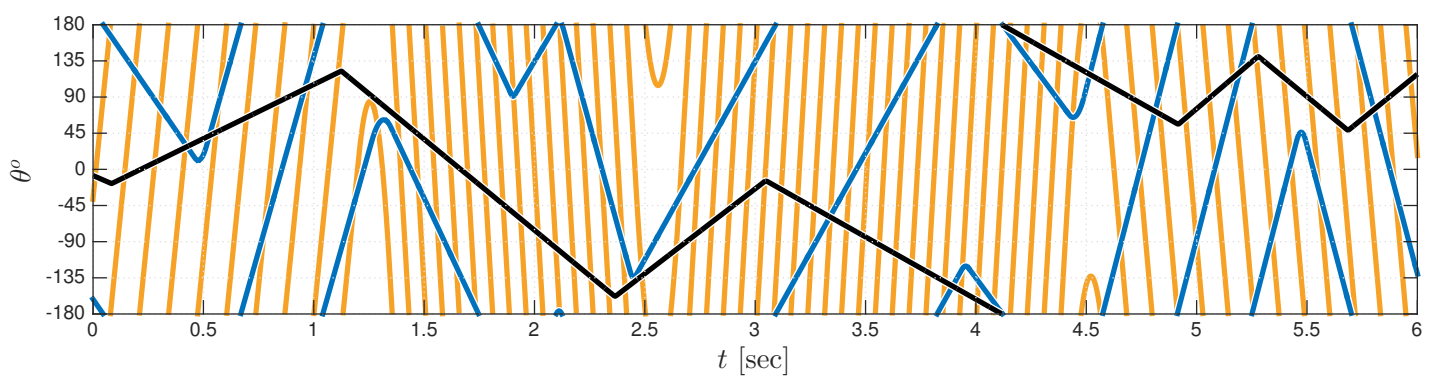

Figure 5. Sample time-series of wing motion for turbulence cases (yellow) B, (blue) C, and (black) D.

mode ' $\mathrm{C}$ ' the produced flow was referred to as ' $1 \mathrm{C}$ '. A sample time-series from the actuation protocol $1 \mathrm{C}$ is provided in Figure 4 for the rods occupying the central $7 M \times 7 M$ of the wind tunnel. The motion of the shear generating wings (flapping) is visibly different from the turbulence generating wings (spinning). The shear generating wings all receive a similar signal that decreases in amplitude across the profile, while the turbulence generating wings perform completely different randomisations of the same base-set of parameters $(t, \omega, a)$.

Measurements were performed in the transverse plane located $x=3.34 \mathrm{~m}=41.3 \mathrm{M}$ from the grid with a rake of four single-wire hot-wires. The wires were made in-house using $5 \mu \mathrm{m}$ diameter tungsten wire mounted to Dantec-style prongs. Sensing lengths of nominally $1 \mathrm{~mm}$ were isolated with a copper plating technique. The wires were operated with a Dantec $54 \mathrm{~N} 82$ multi-channel constant temperature anemometer at an overheat ratio of 1.8. Calibrations were performed in-situ with the grid set to its fully open position using a fourth-order polynomial and 15 reference velocities. An anolog filter was set at $10 \mathrm{kHz}$ and data were sampled at $20.5 \mathrm{kHz}$. Samples were acquired for a minimum of 6 minutes, resulting in 18,000 integral time scales along the centreline for the worst case. The acquired signals were recursively filtered at updated estimates of $1.1 f_{K}$, where $f_{K}=U / 2 \pi \eta$ is the Kolmogorov frequency, in order to reduce noise associated with over-sampling the noise-floor [29]. The Kolmogorov frequency, $f_{K}$, is dependent on the Kolmogorov microscale, which represents the approximate size of the smallest scales in the flow, and is given by,

$$
\eta=\frac{\nu^{3 / 4}}{\langle\epsilon\rangle^{1 / 4}}
$$

where $\langle\epsilon\rangle$ is the mean dissipation rate of turbulent kinetic energy (referred to simply as 'dissipation'). The dissipation in-turn was estimated from the isotropic assumption,

$$
\langle\epsilon\rangle=15 \nu\left\langle\left(\frac{\partial u}{\partial x}\right)^{2}\right\rangle .
$$

In this study, all spatial gradients were estimated with Taylor's frozen flow hypothesis, $(\partial / \partial t)=U(\partial / \partial x)$, and a sixthorder centred-difference scheme. The latter is recommended as a balance between high-wavenumber accuracy and noise filtering for hot-wire anemometry [30]. 
Hot-wire anemometry is often used for wind tunnel studies such as this because it offers good spatial and temporal resolution of the relevant scales in the flow. The absolute uncertainty of this technique, including contributions from the calibration procedure is typically on the order of $\sim 3 \%$ of the velocity measurement [31]. In addition to this, the statistical convergence of a given measurement is dependent on the length scales present in the flow, the sample time, and the specific quantity being measured. In the present study, the integral scale varies from case-to-case, however, in the worst-case $u^{\prime}$ is converged to within $\pm 2 \%$ for the $95 \%$ confidence interval [32]. These uncertainties are contained within the symbols size on the figures of the mean velocity and the velocity fluctuations.

The four hot-wires were mounted to a rake that was traversed along the $z$-direction. The wires were separated by 25, 75 and $225 \mathrm{~mm}$ from the first wire. The majority of the measurements were performed between $z=-0.31 \mathrm{~m}$ and $z=+0.34 \mathrm{~m}$, where $z=0$ is located at the centre of the cross-section. Additional homogeneity scans were performed with the rake rotated to be parallel with the $y$-axis, and then traversed in the $z$-direction between $z=-0.25 \mathrm{~m}$ and $z=+0.29 \mathrm{~m}$ at several different $y$-offsets so that the scans spanned $y=-0.20 \mathrm{~m}$ to $y=+0.22 \mathrm{~m}$. All measurements were performed with a centreline velocity of $U_{0} \approx 10 \mathrm{~m} / \mathrm{s}$, resulting in $R e_{M}=U_{0} M / \nu \approx 52,000$ for all cases.

\section{GENERAL RESULTS}

Mean velocity and turbulence intensity profiles for the 16 flows, grouped by their common shear modes, are presented in Figure 6. In general, the targeted range for these flows was $-2 \leq z / M \leq+2$. A $4 M \times 4 M$ box represents a region that is $36 \%$ of the tunnel width and $54 \%$ of the tunnel height. A model, of for instance a wind turbine, that occupied this entire envelope would cause blockage and pressure drop issues of its own in the present facility, and as a result all such models will be smaller than this envelope. As such, this investigation region represents both the flow that would interact with a model and the flow surrounding it.

The homogeneity of the mean velocity is illustrated in Figure 7 for cases 1A and 4D, which represent the extremes of the flow profiles produced, and are thus representative of the homogeneity throughout this study. The locations of the discrete point measurements are identified in the figure, and the contours are produced with 2D linear interpolation from the non-uniform grid of measurements. The area of the $4 M \times 4 M$ investigation box is highlighted along with the area that could be occupied by a $D=3 M$ model turbine. The homogeneity scan ranges $-2.5 \leq y / M \leq+2.7$ and $-3.1 \leq z / M \leq+3.6$, covering an area of $5.2 M \times 6.7 M$ of the $7.4 M \times 11.1 M$ wind tunnel cross-section. This is sufficient because the boundary layers in this facility for similar levels of turbulence intensity are known to be $1.4 \lesssim \delta / M \lesssim 2.1$ [28], and the homogeneity measurement area thus encompasses the central section of the flow just outside (or inside the end) of the boundary layers. The mean velocity $y$-homogeneity is strong for both cases over the investigation box, and thus the centreline is a representative profile for the whole window.

Delving deeper into the produced flows, the shear mode 1 cases can be taken as an illustrative example. They are shown in Figure 6(a) and have approximately constant mean shear $(\partial U / \partial z)$ and turbulence intensity $\left(u^{\prime} / U\right)$ across the investigation domain. The mean velocity variations are approximately linear and the turbulence intensity is constant to 


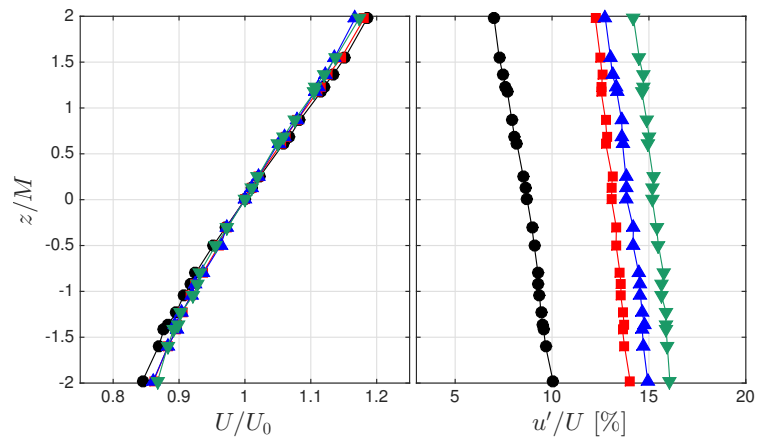

(a)

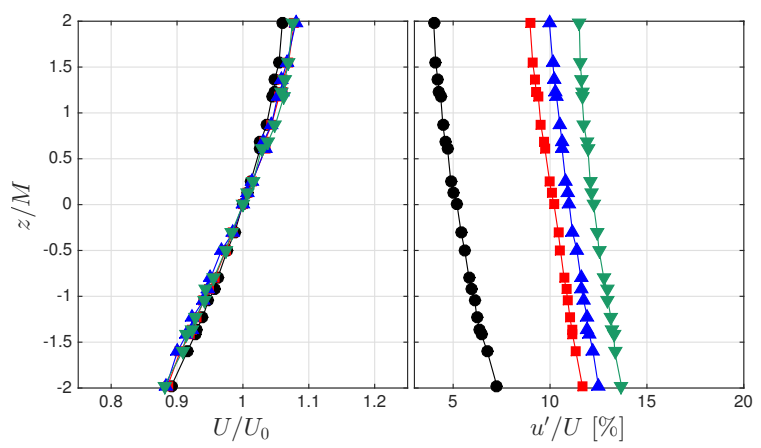

(c)

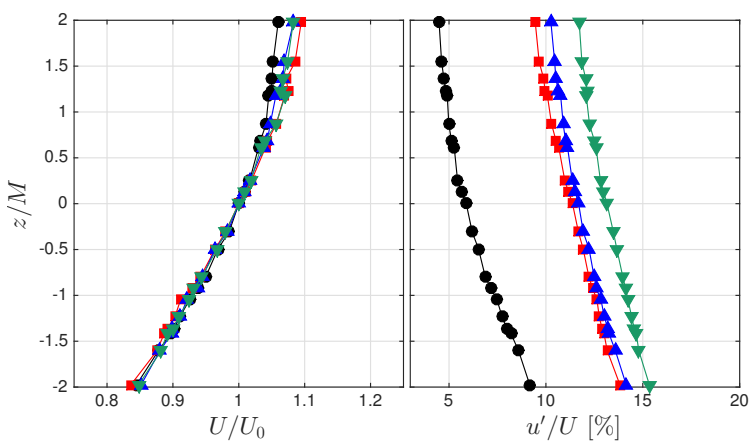

(b)

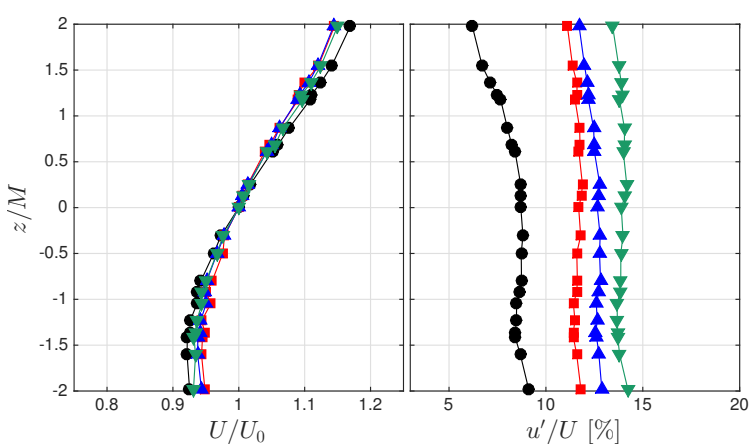

(d)

Figure 6. Mean velocity and turbulence intensity profiles for all cases. Shear generating modes: (a) 1, (b) 2, (c) 3, and (d) 4. Turbulence generating modes: $(\bullet) A,(\square) B,(\Delta) C$, and $(\nabla) D$.
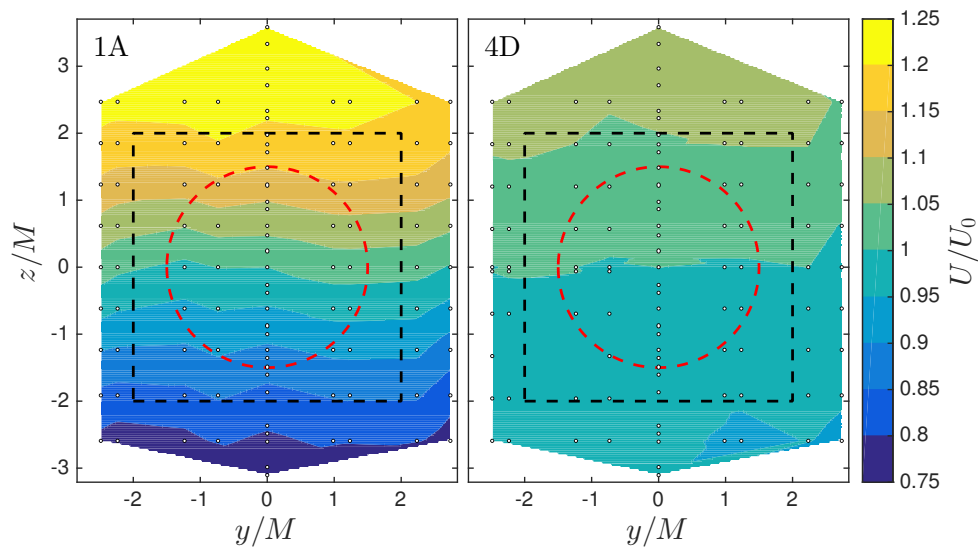

Figure 7. Contours of the mean velocity homogeneity for cases $1 \mathrm{~A}$ and $4 \mathrm{D}$, which represent contrasting extremes of the parameter space and are thus representative. The indicated points are measurement locations. The dashed black box indicates the interrogation window used throughout this work. The red circle identifies the area of the simulated wind turbine with $D=3 M$. The wind tunnel walls are located at $\pm 3.7 M$ in the $y$-direction, and $\pm 5.6 M$ in the $z$-direction.

within $\pm 1.2 \%$ in the worst case $(1 \mathrm{~A})^{*}$. This is explicitly quantified in Table III. A near constant turbulence intensity across

${ }^{*}$ All changes in turbulence intensity are expressed in percent point change to $u^{\prime} / U \times 100$. For example, a $+1.5 \%$ change to $u^{\prime} / U=10 \%$ is $u^{\prime} / U=11.5 \%$. 
the shear simulates the flow assumed by the IEC standard with its single point measurement, i.e., the turbulence intensity is constant across the turbine. As such, these cases represent four different flows where the mean shear is functionally the same but the turbulence intensity increases. This was done by maintaining the same shear operational mode (' 1 ') while adjusting the turbulence mode ('A', 'B', 'C', 'D'). The flows in Figure 6(a) may also be useful for high $R e_{\lambda}$ studies of homogeneous shear flows as the variation in $u^{\prime} / U_{0}$ is comparable to that in $u^{\prime} / U$ (not shown here), and still well below the 7\% peak-to-peak change in $u^{\prime} / U$ in the homogeneous shear flow approximated by Shen and Warhaft [25]. Moreover, the advantage for the investigation of homogeneous shear flows of the present configuration is that the $R e_{\lambda}$ has been changed from 466 to 749 along the centreline while maintaining approximately the same $\partial U / \partial z$. It is also significant that the velocity range across the investigation region achieved here exceeds $U_{0} \pm 15 \%$ for many cases, which is representative of over $61 \%$ of the profiles measured at the Høvsøre site reported by Wagner et al. [8]. Furthermore, the range of turbulence intensities reached here spans $4.6 \% \leq u^{\prime} / U \leq 16.0 \%$, which is well aligned with the most common observations of turbulence intensity at GROWIAN reported by Mücke et al. [12].

The shear mode 2, 3, and 4 cases roughly follow similar trends to those of shear mode 1, i.e., the general mean velocity shear shape is set by the shear mode, and the turbulence is changed by the turbulence mode. This demonstrates that the methodology of setting a shear profile with the vertical wings flapping and the turbulence intensity with the horizontal wings in fully random mode is both transferable to other profiles and repeatable. Some cases present better approximations of constant turbulence intensity compared to others; shear mode 4 appears to produce the best approximation of constant turbulence intensity. In all cases, turbulence mode A produced significantly lower levels of $u^{\prime} / U$. This is because the turbulence generating wings are stationary in mode A, and the turbulence is produced solely by the flapping motion of the shear generating wings. The turbulence intensity in mode $\mathrm{A}$ is thus highly dependent on the motion of the shear wings and is rarely linear. For these modes, the turbulence intensity in the high-speed regions tends to be lower than in the low-speed regions. This is likely a combined effect of having similar fluctuations but a larger local mean velocity, and the wings upstream of the high-speed region having a smaller amplitude of oscillation.

The shear for each case is parameterised in Table III as $\alpha$ from (6), which is typical for wind turbine flows. To determine $\alpha$, we first take the derivative of (6) relative to $z$,

$$
\frac{\partial U}{\partial z}=\frac{U_{H} \alpha}{z}\left(\frac{z}{H}\right)^{\alpha}
$$

where we have also set the reference position to be at a wind turbine hub height, $H$, therefore, $U_{r}=U_{H}$ and $z_{r}=H$. If the derivative is then taken at $z=H$ and both sides are multiplied by $D / U_{H}$, then (10) reduces to

$$
\frac{D}{U_{H}}\left(\frac{\partial U}{\partial z}\right)_{z=H}=\frac{\alpha}{(H / D)}
$$

where $D$ is the wind turbine diameter. To estimate $\alpha$, we assume an artificial turbine diameter of $D=3 M$ and a hub height-to-diameter ratio of $H / D=1$. Values of $H / D$ for real wind turbines range $0.8 \lesssim H / D \lesssim 1.2$, however, $H / D=1$ 
Table III. Flow characteristics for all operational modes. For each case, the measured values are provided at $z / M=-2,0,+2$, from top to bottom, respectively.

\begin{tabular}{|c|c|c|c|c|c|c|c|c|c|}
\hline Mode & $U / U_{0}$ & $\begin{array}{c}u^{\prime} / U \\
{[\%]}\end{array}$ & $R e_{\lambda}$ & $\alpha$ & $\mathcal{K}_{S} / \mathcal{K}_{H}$ & $\mathcal{K} / \mathcal{K}_{H}$ & $L / M$ & $\begin{array}{c}\lambda / M \\
\times 10\end{array}$ & $\begin{array}{l}\eta / M \\
\times 10^{3}\end{array}$ \\
\hline \multirow{3}{*}{$1 \mathrm{~A}$} & 0.84 & 9.7 & 482 & - & - & - & $\begin{array}{l}1.8 \\
\end{array}$ & 1.1 & 2.6 \\
\hline & 1.00 & 8.7 & 466 & 0.28 & 1.02 & 1.04 & 1.8 & 1.0 & 2.4 \\
\hline & 1.19 & 7.3 & 444 & - & - & - & 2.3 & 1.0 & 2.5 \\
\hline \multirow{3}{*}{ 1B } & 0.86 & 13.7 & 587 & - & - & - & 1.9 & 0.9 & 1.9 \\
\hline & 1.00 & 13.1 & 599 & 0.25 & 1.02 & 1.08 & 1.7 & 0.9 & 1.8 \\
\hline & 1.18 & 12.5 & 685 & - & - & - & 2.1 & 0.9 & 1.8 \\
\hline \multirow{3}{*}{$1 \mathrm{C}$} & 0.86 & 14.7 & 641 & - & - & - & 2.0 & 0.9 & $\begin{array}{l}1.8 \\
\end{array}$ \\
\hline & 1.00 & 13.9 & 663 & 0.25 & 1.02 & 1.08 & 1.9 & 0.9 & 1.7 \\
\hline & 1.17 & 13.0 & 749 & - & - & - & 2.3 & 0.9 & 1.7 \\
\hline \multirow{3}{*}{ 1D } & 0.87 & 16.0 & 703 & - & - & - & 2.2 & 1.0 & 1.9 \\
\hline & 1.00 & 15.2 & 749 & 0.25 & 1.02 & 1.09 & 2.0 & 1.0 & 1.8 \\
\hline & 1.17 & 14.5 & 880 & - & - & - & 2.7 & 1.0 & 1.8 \\
\hline \multirow{3}{*}{$2 \mathrm{~A}$} & 0.84 & 8.6 & 449 & - & - & - & 1.8 & 1.2 & 2.8 \\
\hline & 1.00 & 5.9 & 313 & 0.17 & 0.99 & 1.00 & 1.8 & 1.0 & 3.0 \\
\hline & 1.06 & 4.6 & 261 & - & - & - & 2.5 & 1.0 & 3.3 \\
\hline \multirow{3}{*}{$2 B$} & 0.84 & 13.2 & 555 & - & - & - & 1.7 & 0.9 & 2.0 \\
\hline & 1.00 & 11.4 & 545 & 0.21 & 1.00 & 1.03 & 1.8 & 0.9 & 2.0 \\
\hline & 1.09 & 9.7 & 517 & - & - & - & 2.0 & 0.9 & 2.1 \\
\hline \multirow{3}{*}{$2 \mathrm{C}$} & 0.85 & 13.6 & 574 & - & - & - & 1.8 & 0.9 & 2.0 \\
\hline & 1.00 & 11.7 & 566 & 0.19 & 0.99 & 1.03 & 1.9 & 0.9 & 2.0 \\
\hline & 1.08 & 10.5 & 585 & - & - & - & 2.2 & 1.0 & 2.1 \\
\hline \multirow{3}{*}{$2 \mathrm{D}$} & 0.85 & 14.8 & 691 & - & - & - & 2.0 & 1.0 & 2.0 \\
\hline & 1.00 & 13.2 & 743 & 0.20 & 1.00 & 1.05 & 2.4 & 1.1 & 2.1 \\
\hline & 1.08 & 11.9 & 796 & - & - & - & 3.5 & 1.2 & 2.2 \\
\hline \multirow{3}{*}{$3 \mathrm{~A}$} & 0.89 & 6.8 & 349 & - & - & - & 1.6 & 1.1 & 3.0 \\
\hline & 1.00 & 5.2 & 278 & 0.13 & 1.00 & 1.00 & 1.8 & 1.0 & 3.2 \\
\hline & 1.06 & 4.1 & 214 & - & - & - & 2.1 & 1.0 & 3.3 \\
\hline \multirow{3}{*}{ 3B } & 0.89 & 11.3 & 500 & - & - & - & 1.6 & 0.9 & 2.1 \\
\hline & 1.00 & 10.2 & 496 & 0.16 & 1.00 & 1.03 & 1.7 & 0.9 & 2.1 \\
\hline & 1.08 & 9.1 & 486 & - & - & - & 1.9 & 1.0 & 2.2 \\
\hline \multirow{3}{*}{$3 \mathrm{C}$} & 0.88 & 12.2 & 536 & - & - & - & 1.8 & 0.9 & 2.0 \\
\hline & 1.00 & 11.0 & 545 & 0.16 & 1.00 & 1.03 & 1.9 & 0.9 & 2.1 \\
\hline & 1.08 & 10.1 & 581 & - & - & - & 2.2 & 1.0 & 2.2 \\
\hline \multirow{3}{*}{ 3D } & 0.88 & 13.3 & 663 & - & - & - & 2.1 & 1.1 & 2.1 \\
\hline & 1.00 & 12.2 & 706 & 0.16 & 1.00 & 1.05 & 2.5 & 1.1 & 2.1 \\
\hline & 1.07 & 11.6 & 781 & - & - & - & 3.7 & 1.2 & 2.2 \\
\hline \multirow{3}{*}{$4 \mathrm{~A}$} & 0.93 & 8.7 & 406 & - & - & - & 1.4 & 1.0 & 2.5 \\
\hline & 1.00 & 8.7 & 497 & 0.22 & 1.03 & 1.05 & 1.7 & 1.1 & 2.5 \\
\hline & 1.17 & 6.7 & 413 & - & - & - & 2.0 & 1.0 & 2.6 \\
\hline \multirow{3}{*}{$4 B$} & 0.95 & 11.6 & 465 & - & - & - & 1.5 & 0.8 & 2.0 \\
\hline & 1.00 & 11.7 & 507 & 0.17 & 1.03 & 1.07 & 1.5 & 0.8 & 1.9 \\
\hline & 1.15 & 11.4 & 587 & - & - & - & 1.8 & 0.9 & 1.9 \\
\hline \multirow{3}{*}{$4 C$} & 0.94 & 12.7 & 533 & - & - & - & 1.7 & 0.9 & 1.9 \\
\hline & 1.00 & 12.7 & 580 & 0.18 & 1.02 & 1.07 & 1.7 & 0.9 & 1.9 \\
\hline & 1.14 & 12.0 & 656 & - & - & - & 2.0 & 0.9 & 1.9 \\
\hline \multirow{3}{*}{ 4D } & 0.93 & 13.8 & 613 & - & - & - & 2.0 & 0.9 & 1.9 \\
\hline & 1.00 & 13.9 & 692 & 0.19 & 1.02 & 1.08 & 2.0 & 1.0 & 1.9 \\
\hline & 1.15 & 13.8 & 871 & - & - & - & 2.7 & 1.1 & 1.9 \\
\hline
\end{tabular}

is a typical value and this parameter acts only to scale $\alpha$. The mean velocity gradient, $(\partial U / \partial z)_{z=H}$, is estimated by fitting a straight line across $D$ and taking the slope. 
An alternative to methodology for quantifying the shear is to use a discretised integral quantity such as $\mathcal{K}_{S}$ from (4), which is also provided in Table III relative to $\mathcal{K}_{H}$. The influence of both turbulence intensity and shear are reflected in the kinetic energy flux that leads to (5). If one replaces $U_{i}$ in (4) with $U_{i}+u_{i}$, then performs a Taylor expansion and neglects the high-order terms, the discretised kinetic energy flux through the turbine area incorporating both the effects of shear and turbulence intensity is

$$
\mathcal{K}=\frac{1}{2} \rho \sum_{i} U_{i}^{3}\left[1+3\left(\frac{u_{i}^{\prime}}{U_{i}}\right)^{2}\right] A_{i} .
$$

This quantity is also provided in Table III relative to $\mathcal{K}_{H}$.

Similar trends are apparent in the shear produced by the various operational modes as quantified in Table III. For all four shear mode cases (' 1 ', '2', '3', '4'), $\alpha$ is the same \pm 0.01 for the cases where the turbulence wings are actuated (' $\mathrm{B}$ ', 'C', 'D'). This illustrates that a similar shear level can be achieved while changing the turbulence intensity. In all cases, $\alpha$ is different for the 'A' turbulence case, which is likely a result of a slower approach to equilibrium due to less transverse transport of turbulent energy because of the lower turbulence levels. Interestingly, the integral quantity $\mathcal{K}_{S} / \mathcal{K}_{H}$ absorbs this difference, and for each shear mode $\mathcal{K}_{S} / \mathcal{K}_{H}$ is constant to within \pm 0.005 , suggesting the bulk effect of not actuating the turbulence wings is marginal. Finally, $\mathcal{K} / \mathcal{K}_{H}$, which incorporates the effect of turbulence shows similar trends for all cases, i.e., it is set by the 'A' mode case, and then grows with turbulence intensity through to the 'D' case.

Several other parameters are also listed in Table III for each case at $z / M=-2,0$, and +2 . For instance, the mean velocity relative to the centreline velocity $\left(U / U_{0}\right)$, the local turbulence intensity $\left(u^{\prime} / U\right)$ and the Taylor microscale Reynolds number $\left(R e_{\lambda}\right)$ are provided. The relevant length scales for the produced flows are also given in Table III. The integral length scale $(L)$ was estimated as the integral under the autocorrelation function to the first zero-crossing. The Taylor microscale is given by,

$$
\lambda=\sqrt{\frac{\left\langle u^{2}\right\rangle}{\left\langle(\partial u / \partial x)^{2}\right\rangle}}
$$

and the final length scale of relevance is the Kolmogorov microscale $(\eta)$ given by (8). These scales represent the approximate size of the large scales $(L)$, intermediate scales $(\lambda)$, and smallest scales $(\eta)$ in the flow, respectively.

The variation in length scales across the shear profiles are quantified in Table III and are plotted in Figure 8 for four illustrative examples. In general, $\eta, \lambda$ and $L$ are relatively constant across $-2 \leq z / M \leq+2$. In particular, the smallest and intermediate scales practically do not vary, while marginally larger variation is exhibited by the large scales. However, variation in the large scales is to be expected to a certain degree given that the advection velocity varies with $z$. Nonetheless, the relative constancy of the length scales in the flow is again a desirable trait for a bespoke flow used to test wind turbine models because the influence of the incoming conditions can be attributed to only the shear and turbulence intensity, which are adjustable independently (to a certain degree), without worry of significant variations in length scales across the flow.

As a final note, we did try to produce linear profiles with different slopes by using a linear change in $\theta$ across the test-section from ranges smaller than $0 \leq \theta \leq 90^{\circ}$ (this is the range employed by shear mode 1 ). However, we found that this increased the instantaneous blockage of the tunnel resulting in a choked flow with extreme pulsations and dangerously unsteady loads on our fan blades. This is the reason that only one shear mode with a linear change in $\theta$ is presented here. 


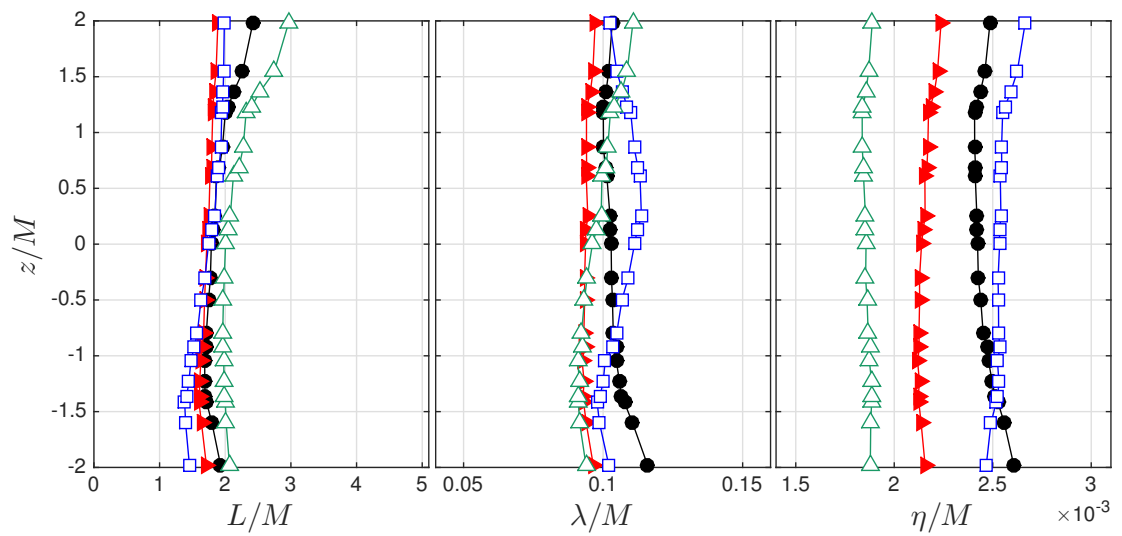

Figure 8. Integral length scale, Taylor microscale and Kolmogorov microscale profiles for cases $(\bullet) 1 A,(\bullet) 3 B,(\square) 4 A$, and $(\triangle)$ 4D.

\section{MATCHING SHEAR AND TURBULENCE INTENSITY}

The results of the previous section can be used to match shear or turbulence intensity in order to decouple their influence on a problem of industrial interest (e.g., a wind turbine). For instance, Figure 6(a) shows that approximately linearly sheared velocity profiles can be reproduced with different levels of turbulence intensity. The centreline turbulence intensity changes from $8.7 \%$ for case $1 \mathrm{~A}$ to $15.2 \%$ for case $1 \mathrm{D}$ while maintaining $\mathcal{K}_{S} / \mathcal{K}_{H}=1.02$. If the preferred parameterisation of the shear is $\alpha$, then the change is smaller $(13.1 \%$ for $1 \mathrm{~B}$ to $15.2 \%$ for $1 \mathrm{D})$. The same is true for the non-linear profiles, i.e., $5.9 \%$ for $2 \mathrm{~A}$ growing to $13.2 \%$ for $2 \mathrm{D}$. Thus, an object, say a building or wind turbine model, could be placed in these flows, with approximately the same mean velocity profile, but significantly different turbulence intensity in order to assess the effect of turbulence intensity in a shear flow independently of the shear itself. This is a problem that has traditionally been very difficult to simulate experimentally because studies investigating the effects of shear have been performed by placing obstructions in the boundary layer of a wind tunnel where turbulence intensity and shear cannot easily be decoupled; see for instance the studies on cubes [19], or wind turbine models [18, 33].

The present set-up also allows for the opposite variation, i.e., the turbulence intensity can be kept relatively constant while the mean shear profile can be changed substantially. Figure 9(a) compares cases $1 \mathrm{~A}$ and 3B. These profiles were chosen based on the produced flow rather than the generation mechanism. The shear producing pattern generates background turbulence which is modified by the turbulence generating wings, and the resulting turbulence intensity is a combined effect of these two protocols. Hence, the turbulence levels of $1 \mathrm{~A}$ and $3 \mathrm{~B}$ are closer than $1 \mathrm{~A}$ and $3 \mathrm{~A}$. The turbulence intensity profiles for cases $1 \mathrm{~A}$ and $3 \mathrm{~B}$ track together and their difference is $\sim 1.5 \%$, which is within the range of the variation of either of the profiles. However, their mean velocity profiles and thus shear, differ significantly, i.e., $\alpha=0.28$ and $\mathcal{K}_{S} / \mathcal{K}_{H}=1.02$ for $1 \mathrm{~A}$ while $\alpha=0.16$ and $\mathcal{K}_{S} / \mathcal{K}_{H}=1.00$ for $3 \mathrm{~B}$. Figure 9 (b) shows another set of three profiles where the centreline turbulence intensity if fixed at $11.35 \% \pm 0.35 \%$, while $0.16 \leq \alpha \leq 0.21$. The proportional change to the shear is much larger than to the turbulence intensity, and this difference is also visible in Figure 9(b). By comparing profiles such as the examples provided in Figure 9, the effect of turbulence intensity is marginallised while the effect of 


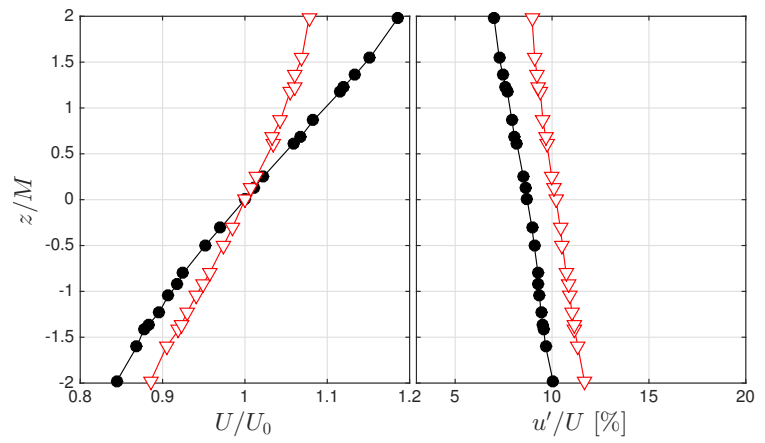

(a)

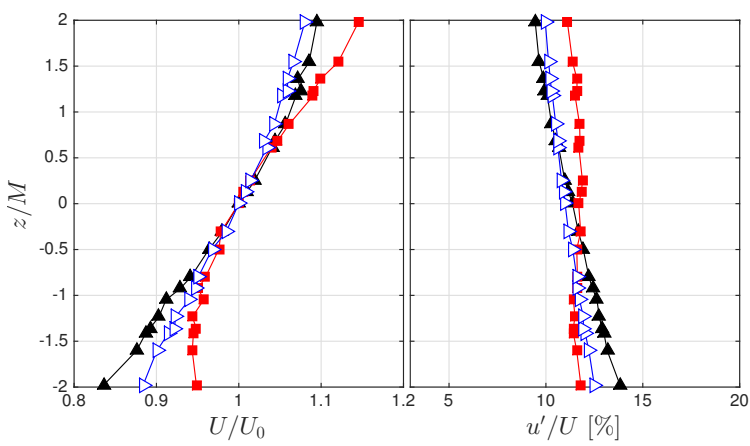

(b)

Figure 9. Mean velocity and turbulence intensity profiles for cases $(\nabla) 1 A,(\mathbf{\Delta}) 2 B,(\bullet) 3 B,(\triangleright) 3 C$, and $(\square) 4 B$. The profiles in (a) have turbulence intensity that trends in the same way and differs by only $1.5 \%$, while $\alpha$ changes from 0.28 to 0.16 . In (b), the centreline turbulence intensity is the same to within $0.7 \%$, while the slope changes in the range $0.16 \leq \alpha \leq 0.21$.

shear is emphasised. Thus, this methodology allows for one to produce flows where shear is kept approximately constant and turbulence intensity is varied, and turbulence intensity is kept approximately constant and shear is varied, allowing for detailed analysis of the influence of shear and turbulence independently. It must be emphasized that conditions such as these have previously been unattainable in experimental facilities.

\section{ANALYSIS OF THE TIME-SERIES INFORMATION}

In addition to producing bespoke profiles of the mean velocity and turbulence intensity, it is also desirable to have velocity spectra and probability density functions (PDFs) that are comparable across the produced shear profiles. When these parameters are similar across the flow field, it implies that the dynamics of the flow are comparable. The one-dimensional streamwise velocity spectra $\left(E_{11}\right.$, where $\left.\left\langle u^{2}\right\rangle=\int_{0}^{\infty} E_{11}(k) \mathrm{d} k\right)$ from cases $1 \mathrm{~A}, 3 \mathrm{~B}, 4 \mathrm{~A}$, and $4 \mathrm{D}$ are presented in Figure 10 normalised by Kolmogorov variables; these cases were found to be representative of the measurements as a whole. Normalisation by Kolmogorov variables collapses the small scales (large wavenumbers). The figure illustrates that in all cases a near (but not exact) $k^{-5 / 3}$ spectrum is achieved and the scaling and dissipative ranges are well collapsed. There is only some minor variation between the cases at the largest scales (lowest wavenumbers). This is most pronounced for cases $1 \mathrm{~A}$ and $4 \mathrm{~A}$, and is a result of the significant blockage produced by the solid wings upstream of the low velocity region of the produced shear. However, both this study and that by Hearst and Lavoie [23] show that these differences do not permeate down the cascade, or into the centre of the test-section. It is also worth noting that the marginal departure from $k^{-5 / 3}$ observed here is in keeping with typical results of turbulence produced in the wake of both regular grids and active grids [34].

The PDFs of the velocity distributions for the same four illustrative cases are given in Figure 11. In general, any difference between the PDFs is restricted to the tails of the distributions and thus represent little impact (rare events) on a model placed in such a flow. Again, the greatest variation is present in an 'A' shear mode case where there is significant 

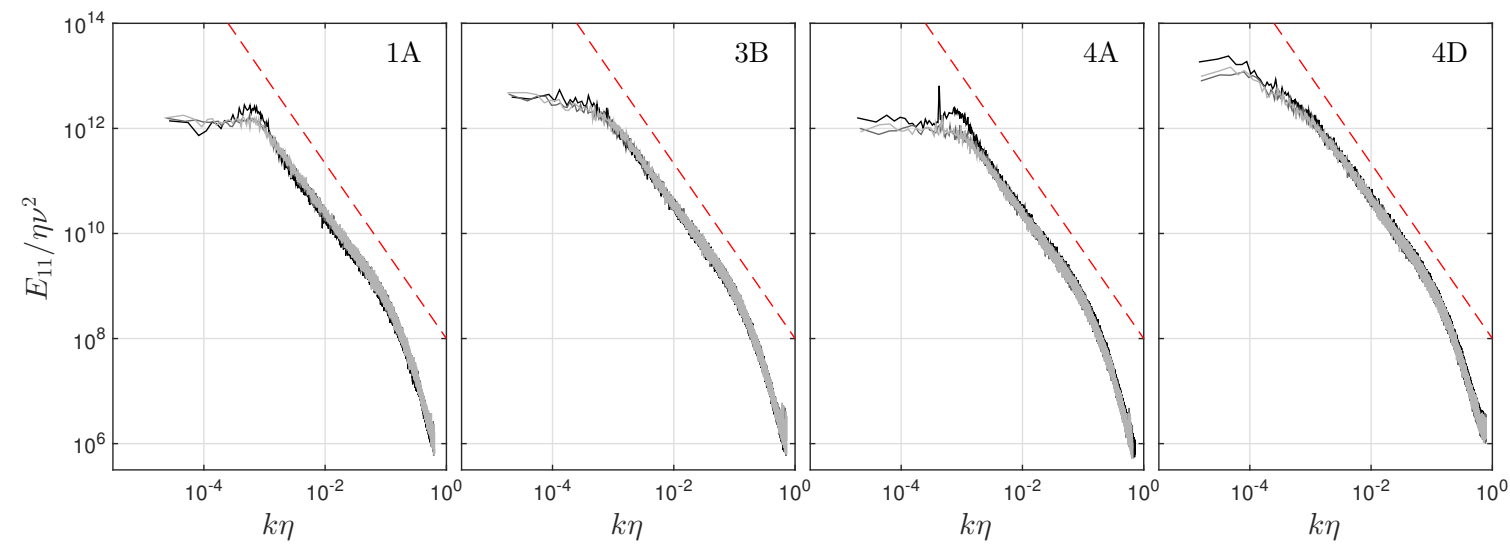

Figure 10. Velocity spectra normalised by Kolmogorov variables for four illustrative cases. In all plots, lines from darkest to lightest represent spectra measured at $z / M=-2,0$, and +2 , respectively. The dashed lines represent $k^{-5 / 3}$.
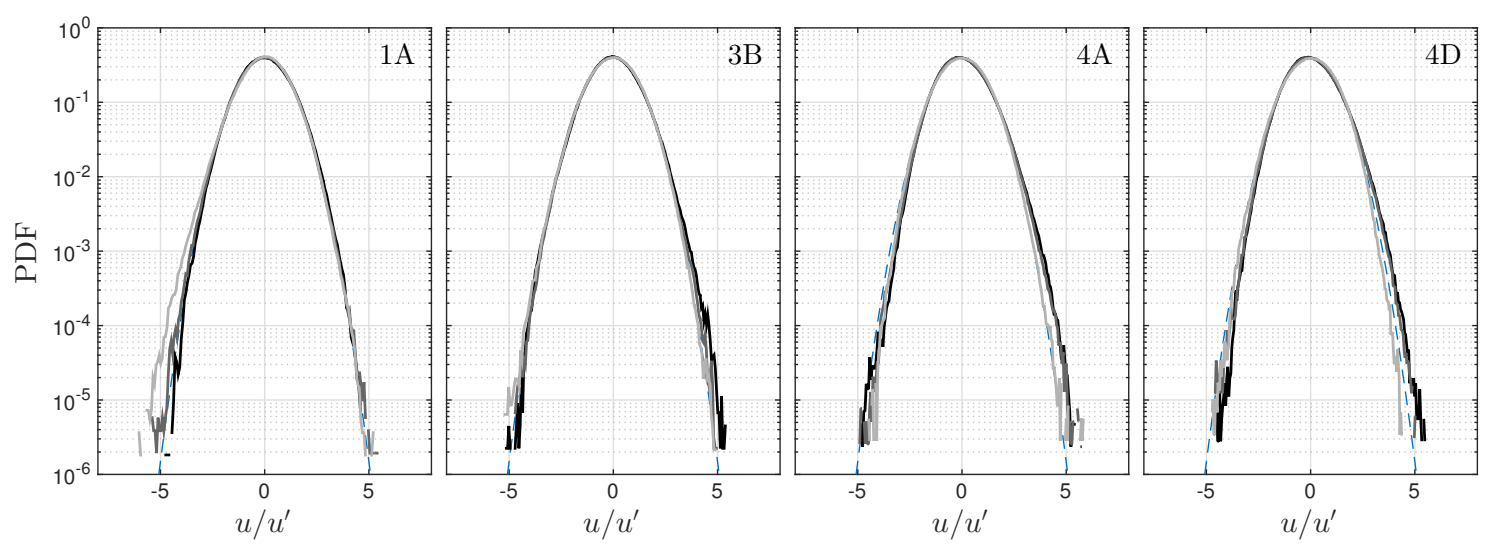

Figure 11. Probability density functions for four illustrative cases. In all plots, lines from darkest to lightest represent spectra measured at $z / M=-2,0$, and +2 , respectively, and the blue dashed line represents a Gaussian distribution.

blockage upstream of the low-speed section of the velocity profile. A Gaussian curve is provided for reference in Figure 11. In many of the sub-figures, the Gaussian curve is barely visible because the measurements are approximately Gaussian. The difference is primarily at the tails, where atmospheric turbulence is known to deviate from a Gaussian distribution [12, 35]. Nonetheless, Berg et al. [35] showed with large eddy simulations that the impact of non-Gaussian effects on wind turbine loads was negligible, in part due to the inherent low-pass filtering of the wind turbine system, which does not have sufficient frequency response to react to these rare and short-lived events.

An alternative way to consider the instantaneous distribution of velocities is through the velocity increments [12], $\Delta u=u(t)-u(t+\gamma T)$, where $T$ is the integral time-scale and $\gamma$ is an arbitrary multiplication factor. The integral timescale is the time analog of the integral length-scale, i.e., it represents the approximate longest time in the signal where the flow is correlated. By considering the velocity increments at various positions, we gain insight on how similar the gusting and lulling behaviour in the flow is at these positions with respect to the time-scale $\gamma T$. In Figure 12, velocity 

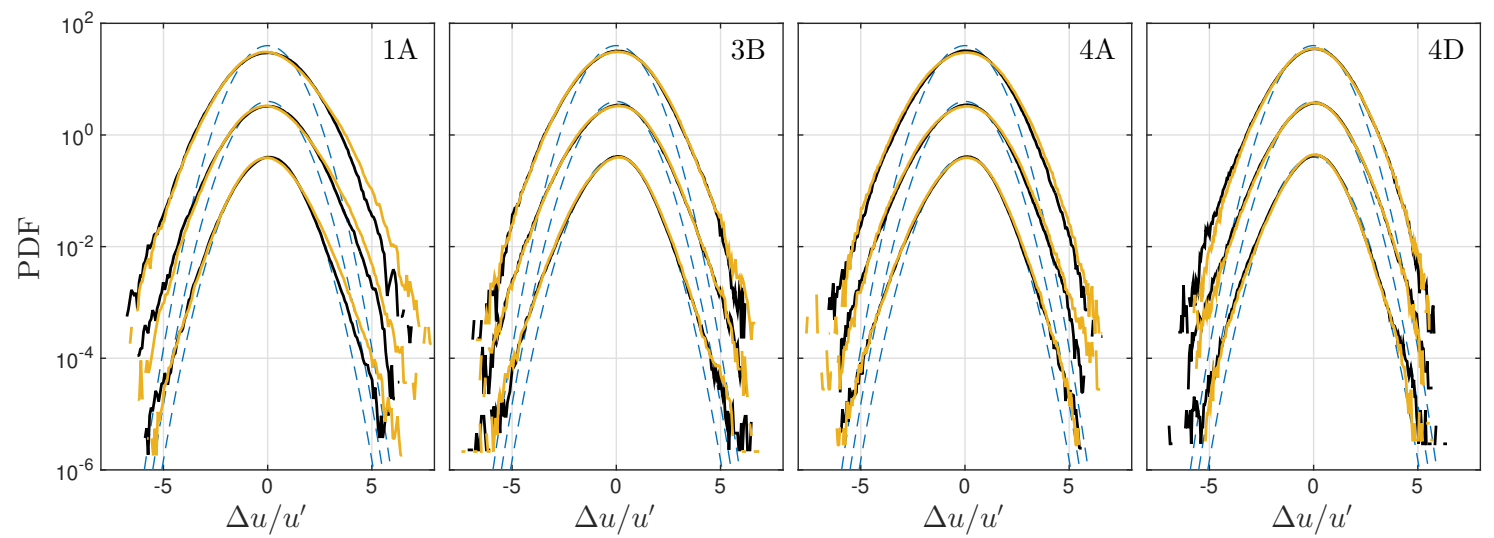

Figure 12. Probability density functions of the velocity increment $(\Delta u=u(t)-u(t+\gamma T))$ for four illustrative cases. In all plots, curves represent increment offsets of $\gamma=1,2$, and 3 from inner most curve to outermost curve, respectively, and the dark line is measured at $z / M=-2$ while the light line is at $z / M=+2$. The blue dashed line represents a Gaussian distribution. The curves illustrate that across the investigation range any changes for these changes in increment range are restricted only to the tails of the PDFs, representing events that occur less frequently.

increments are shown for our four illustrative cases at three different increment levels, $1 T, 2 T$ and $3 T$. Integer increments of $T$ were chosen such that the increment was always performed across velocity signals that were part of different large scale events (or, on very rare occasions, on the extreme edges of a single event). Curves are compared from $z / M=-2$ and +2 , representing the two edges of the interrogation area. There is typically good agreement between the illustrated PDFs of the velocity increments. The tails of the PDFs of velocity increments are always above the Gaussian curve, which agrees with the measurements reported by Mücke et al. [12] from GROWAIN. In our simulated flows, the largest deviations again occur for the 'A' cases near the tails, which suggests the flow is marginally more uniform when it is supplemented with additional background turbulence. Nonetheless, the velocity increment PDFs suggest that the gusting and lulling motions of the flow are comparable across the entire investigation domain, which is again a desirable characteristics for model testing.

\section{DISCUSSION}

The approach described herein is of significant value to the wind engineering community as it provides a methodology to produce flows where the mean shear can be held relatively constant and the turbulence intensity can be changed, and where the turbulence intensity can be held constant and the mean shear changed. This allows for these two parameters to be decoupled, and their influence investigated separately. This is of particular relevance for investigations of the wake of obstacles, e.g., cubes, where the effects of turbulence have been isolated from the effects of shear [27], but there is seemingly no way to perform the opposite investigation. It is also of use to the wind turbine community who have used grids to investigate the influence of free-stream turbulence on wind turbines and blades [36, 37, 38], and placed wind turbines in boundary layers to produce shear [18, 33], but have no way to isolate these parameters in a turbulent shear flow. 


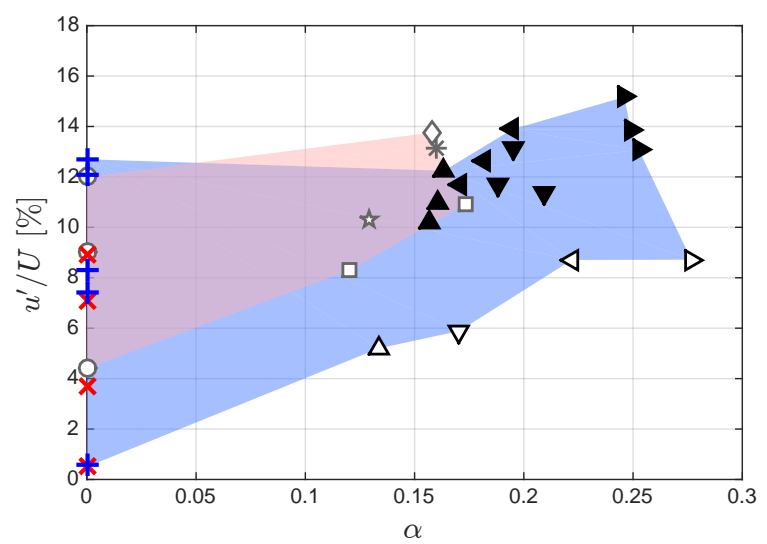

Figure 13. Parameter space of wind tunnel turbulent shear flows. Measurements from the present study are represented by the black triangles: $(\triangleright)$ shear mode $1,(\nabla)$ shear mode $2,(\triangle)$ shear mode 3 , and $(\triangleleft)$ shear mode 4 ; the empty triangles represent the turbulence mode A measurements. Homogeneous turbulence $(\alpha=0)$ cases from the same grid are also included from $(\times)$ Ref. [27], and (+) Ref. [28]. Measurements from other wind turbine model studies in wind tunnels are presented as gray symbols: ( $\square$ ) Ref. [18], $(\diamond)$ Ref. [15], ( $\succsim)$ Ref. [17], $(\bigcirc)$ Ref. [37], and $(*)$ Ref. [39]. The red shaded area represents the parameter space of wind tunnel experiments relying on the wall to produce the shear. The blue shaded region represents the parameter space occupied by the presented methodology.

Returning to the equivalent wind speed provided in (5), this facility allows for the variation of all parameters on the righthand side of the equation. While this work did not particularly focus on changing the incoming flow angle, $\phi$, this could easily be added to the experimental system by testing the turbine at different incident angles. Recall that (3) and (5) are directly related to the kinetic energy flux through the turbine's swept area, and that this is in turn proportional to the power output of the turbine. It would thus be particularly interesting to see if the produced power from cases $1 \mathrm{C}\left(\mathcal{K} / \mathcal{K}_{H}=1.08\right.$, $\left.\alpha=0.25, u_{0}^{\prime} / U_{0}=13.9 \%\right)$ and $4 \mathrm{D}\left(\mathcal{K} / \mathcal{K}_{H}=1.08, \alpha=0.19, u_{0}^{\prime} / U_{0}=13.9 \%\right)$, which differ only in the shape of the profile is measurably different. Conversely, comparison of cases $1 \mathrm{~B}\left(\mathcal{K} / \mathcal{K}_{H}=1.08, \alpha=0.25, u_{0}^{\prime} / U_{0}=13.1 \%\right)$ and $1 \mathrm{D}$ $\left(\mathcal{K} / \mathcal{K}_{H}=1.09, \alpha=0.25, u_{0}^{\prime} / U_{0}=15.2 \%\right)$ allows for investigation of similar kinetic energy flux and shear with different turbulence intensity. In addition to comparisons such as these, the methodology presented here more simply allows access to a significantly larger portion of the parameter space in which wind turbines operate than accessible by traditional means. Figure 13 shows how the present cases lie within the $\left(\alpha, u^{\prime} / U\right)$ parameter space and compares it to previous tests conducted on wind turbine models in turbulent and shear flows. Data were digitized from the other studies presented in Figure 13 and the hub height was used as the reference position for the least-squares fit to (6). As identified in section 1, measurements ahead of real wind turbines show that this parameter space spans $0 \% \lesssim u^{\prime} / U \lesssim 40 \%$ [12] and $-0.2 \lesssim \alpha \lesssim 0.35[11,8]$. When combined with measurements of the possible homogeneous and isotropic turbulent profiles produced by this grid $[28,27]$, it is clear that a significantly larger portion of the wind turbine's operational parameter space is now accessible compared to the methods presently in use. The space can also be further expanded in the future by implemented new shear modes and increasing the turbulence intensity; the latter could be done by slowing the rotation of the wings or increasing $U_{0}[23]$. 
Future work should focus on exploiting the techniques described herein to investigate the impact of turbulence and shear (independently) on the instantaneous loads experienced by a wind turbine as well as the evolution of the wake. Both of these were previously inaccessible to experimentalists without the present methodology.

\section{CONCLUSIONS AND SUMMARY}

An active grid was used to generate 16 different turbulent shear flows. This was done by programming the vertical bars to flap through varying angles across the span of the wind tunnel cross-section to create a variation in the blockage across the test-section. The horizontal bars were programmed to operate in a fully random fashion where variation in the input to these bars was used to change the background turbulence levels in the shear flows. Using this approach, it was demonstrated that turbulent shear profiles could be produced that had remarkably similar mean velocity profiles, but markedly different turbulence levels. It was also demonstrated that the reverse could be achieved, i.e., that two flows with very similar turbulence intensity levels and profiles could be produced with very different mean velocity profiles and thus shear. The produced flows have centreline turbulence intensities that span $5.2 \% \leq u_{0}^{\prime} / U_{0} \leq 15.2 \%$, and power-law shear parameters ranging $0.13 \leq \alpha \leq 0.28$. This provides significantly improved coverage of the parameter space in which real wind turbines operate compared to traditional methodologies that use or modify the naturally occurring boundary layer on a wind tunnel wall. Velocity spectra and probability density functions were used to illustrate that the dynamics of the flow do not significantly differ across the investigated domain. Finally, with the growing prominence of active grids in the fluid mechanics and atmospheric sciences research communities, it is likely many groups already possess the ability to utilise these techniques and perform such investigations.

\section{ACKNOWLEDGEMENTS}

The authors acknowledge the financial support of the European Research Council (ERC Grant agreement No. 277472), and the Engineering and Physical Sciences Research Council of the United Kingdom (EPSRC Grant Ref. No. EP/I037717/1). RJH acknowledges the financial support of the Natural Sciences and Engineering Research Council of Canada (NSERC). BG acknowledges the financial support of the Leverhulme Trust.

\section{REFERENCES}

1. Vanderwel C, Tavoularis S. Coherent structures in uniformly sheared turbulent flow. J. Fluid Mech. 2011; 689:434464.

2. Frehlich R, Meillier Y, Jensen ML, Balsley B. A statistical description of small-scale turbulence in the low-level nocturnal jet. J. Atmos. Sci. 2004; 61:1079-1085. 
3. Piper M, Lundquist JK. Surface layer turbulence measurements during a frontal passage. J. Atmos. Sci. 2004; 61:1768-1780.

4. Lavoie P, Djenidi L, Antonia RA. Effects of initial conditions in decaying turbulence generated by passive grids. $J$. Fluid Mech. 2007; 585:395-420.

5. Krogstad PA, Davidson PA. Freely decaying, homogeneous turbulence generated by multi-scale grids. J. Fluid Mech. 2011; 680:417-434.

6. Hearst RJ, Lavoie P. Effects of multi-scale and regular grid geometries on decaying turbulence. J. Fluid Mech. 2016; 803:528-555.

7. IEC 61400-12-1. Power performance measurements of electricity producing wind turbines 2005.

8. Wagner R, Antoniou I, Pedersen SM, Courtney MS, Jørgensen HE. The influence of the wind speed profile on wind turbine performance measurements. Wind Energ. 2009; 12:348-362.

9. Wagner R, Courtney M, Gottschall J, Lindelöw-Marsden P. Accounting for the speed shear in wind turbine power performance measurements. Wind Energ. 2011; 14:993-1004.

10. Choukulkar A, Pichugina Y, Clack CTM, Calhoun R, Banta R, Brewer A, Hardesty M. A new formulation for rotor equivalent wind speed for wind resource assessment and wind power forecasting. Wind Energ. 2016; 19:1439-1452.

11. Dimitrov N, Natarajan A, Kelly M. Model of wind shear conditional on turbulence and its impact on wind turbine loads. Wind Energ. 2015; 18:1917-1931.

12. Mücke T, Kleinhans D, Peinke J. Atmospheric turbulence and its influence on the alternating loads on wind turbines. Wind Energ. 2011; 14:301-316.

13. Counihan J. An improved method of simulating an atmospheric boundary layer in a wind tunnel. Atmos. Environ. 1969; 3:197-214.

14. Counihan J. Simulation of an adiabatic urban boundary layer in a wind tunnel. Atmos. Environ. 1973; 7:673-689.

15. Cal RB, Lebrón J, Castillo L, Kang HS, Meneveau C. Experimental study of the horizontally averaged flow structure in a model wind-turbine array boundary layer. J. Renewable Sustain. Energy 2010; 2(013106).

16. Taddei S, Manes C, Ganapathisubramani B. Characterisation of drag and wake properties of canopy patches immersed in turbulent boundary layers. J. Fluid Mech. 2016; 798:27-49.

17. Bossuyt J, Howland MF, Meneveau C, Meyers J. Measurement of unsteady loading and power output variability in a micro wind farm model in a wind tunnel. Exp. Fluids 2017; 58(1).

18. Chamorro LP, Porté-Agel F. A wind tunnel investigation of wind-turbine wakes: boundary-layer turbulence effects. Boundary-Layer Meteorol 2009; 132:129-149.

19. Castro I, Robins A. The flow around a surface-mounted cube in uniform and turbulent streams. J. Fluid Mech. 1977; 79(2):307-335.

20. Nedić J, Tavoularis S. Energy dissipation scaling in uniformly sheared turbulence. Phys. Rev. E 2016; 93(3):033 115.

21. Makita H. Realization of a large-scale turbulence field in a small wind tunnel. Fluid Dyn. Res. 1991; 8:53-64. 
22. Larssen JV, Devenport WJ. On the generation of large-scale homogeneous turbulence. Exp. Fluids 2011; 50:12071223.

23. Hearst RJ, Lavoie P. The effect of active grid initial conditions on high Reynolds number turbulence. Exp. Fluids 2015; 56(10): 185

24. Bodenschatz E, Bewley GP, Nobach H, Sinhuber M, Xu H. Variable density turbulence tunnel facility. Rev. Sci. Inst. 2014; 85(093908).

25. Shen $X$, Warhaft $Z$. The anisotropy of the small scale structure in high Reynolds number $\left(R_{\lambda} \sim 1000\right)$ turbulent shear flow. Phys. Fluids 2000; 12(11):2976-2989.

26. Cekli HE, van de Water W. Tailoring turbulence with an active grid. Exp. Fluids 2010; 49:409-416.

27. Hearst RJ, Gomit G, Ganapathisubramani B. Effect of turbulence on the wake of a wall-mounted cube. J. Fluid Mech. 2016; 804:513-530.

28. Dogan E, Hanson R, Ganapathisubramani B. Interactions of large-scale free-stream turbulence with turbulent boundary layers. J. Fluid Mech. 2016; 802:79-107.

29. Mi J, Deo RC, Nathan GJ. Fast-convergence iterative scheme for filtering velocity signals and finding Kolmogorov scales. Phys. Rev. E 2005; 71(066304).

30. Hearst RJ, Buxton ORH, Ganapathisubramani B, Lavoie P. Experimental estimation of fluctuating velocity and scalar gradients in turbulence. Exp. Fluids 2012; 53(4):925-942.

31. Jørgensen FE. How to measure turbulence with hot-wire anemometers - A practical guide. Dantec Dynamics 2002.

32. Benedict LH, Gould RD. Towards better uncertainty estimates for turbulence statistics. Exp. Fluids 1996; 22:129136.

33. Howard KB, Guala M. Upwind preview to a horizontal axis wind turbine: a wind tunnel and field-scale study. Wind Energ. 2016; 19:1371-1389.

34. Mydlarski L, Warhaft Z. On the onset of high-Reynolds-number grid-generated wind tunnel turbulence. J. Fluid Mech. 1996; 320:331-368.

35. Berg J, Natarajan A, Mann J, Patton EG. Gaussian vs non-Gaussian turbulence: impact on wind turbine loads. Wind Energ. 2016; 19:1975-1989.

36. Devinant P, Laverne T, Hureau J. Experimental study of wind-turbine airfoil aerodynamics in high turbulence. J. Wind Eng. Ind. Aero. 2002; 90:689-707.

37. Sicot C, Devinant P, Laverne T, Loyer S, Hureau J. Experimental study of the effect of turbulence on horizontal axis wind turbine aerodynamics. Wind Energ. 2006; 9:361-370.

38. Maldonado V, Castillo L, Thormann A, Meneveau C. The role of free stream turbulence with large integral scale on the aerodynamic performance of an experimental low Reynolds number S809 wind turbine blade. J. Wind Eng. Ind. Aero. 2015; 142:246-257.

39. Aubrun S, Loyer S, Hancock PE, Hayden P. Wind turbine wake properties: Comparison between a non-rotating simplified wind turbine model and a rotating model. J. Wind Eng. Ind. Aero. 2013; 120:1-8. 